\title{
A posteriori error analysis of a domain decomposition algorithm for unilateral contact problem
}

\author{
L. Gallimard ${ }^{\mathrm{a}, *}$, T. Sassi ${ }^{\mathrm{b}}$ \\ a Laboratoire Energétique, Mécanique, Electromagnétisme, Université Paris Ouest Nanterre La Défense, 50 rue de Sèvres, 92410 Ville d'Avray, France \\ ${ }^{\mathrm{b}}$ Laboratoire de Mathématiques Nicolas Oresme, Université de Caen BP 5186, F 14032 Caen Cedex, France
}

\begin{abstract}
A B S T R A C T
In this paper, we consider a domain decomposition algorithm associated to a finite element method to approximate a unilateral frictionless contact problem between two elastic bodies. We present a global error estimator that takes into account of the error introduced by finite element analysis as well as the error introduced by the iterative resolution of the domain decomposition algorithm. The control of these error sources is a key point in order to introduce adaptive techniques and we propose error indicators that estimate the contribution of each source of error.
\end{abstract}

Keywords:

Error estimation

Domain decomposition

Unilateral contact

Discretization error indicator

Algorithm error indicator

\section{Introduction}

Multi-body contact problems are frequent in structural analysis. They are characterized by inequality constraints such as nonpenetration conditions, sign condition on the normal constraints, and an active contact zone which is unknown a priori. Several approaches exist for solving the non-linear equations issued from the finite element discretization of frictionless contact problems $[1,2]$. An important point is to evaluate the approximation errors introduced by the numerical algorithm. For contact problems two sources of errors are introduced, the first one due to the spatial discretization (the finite element mesh), the second one due to the algorithm used to solve the non-linear equations. Here, we consider a natural domain decomposition which consists in adapting a Neumann-Dirichlet method to a multi-body frictionless contact problem by preserving the physical decomposition of the structure $[3,4]$.

Several methods have been developed over many years to evaluate the global quality of FE analysis. For linear problems the earlier works have lead to estimators based on the residual of the equilibrium equation [5], estimators based on the concept of constitutive relation [6], and estimators using the smoothing of the finite element stresses [7]. For multi-body contact problems there is much less work [8-14].

\footnotetext{
* Corresponding author.

E-mail addresses: laurent.gallimard@u-paris10.fr (L. Gallimard), sassi@math. unicaen.fr (T. Sassi).
}

The objective of this paper is to present an a posteriori global error estimator for a frictionless multi-body contact problem solved by a Neumann-Dirichlet decomposition algorithm [3], assuming small strains and displacements. Additionally, two error indicators that allow to estimate the part of the error due to the spatial discretization and that due to the domain decomposition algorithm are developed. We present, in this paper, a first application for six-node triangular elements with matching meshes on the contact zone.

The paper is organized as follows: in Section 2, we introduce the frictionless contact problem to be solved. The domain decomposition algorithm is described in Section 3. In Section 4, the continuous and the finite element variational formulations are introduced. Section 5 is devoted to the formulation of the global error estimator, the discretization error indicator and the algorithm error indicator. Finally, in Section 6 the different errors are analyzed through numerical examples.

\section{Reference problem description}

We consider the problem of two bidimensional elastic bodies $\Omega^{1}$ and $\Omega^{2}$ under the assumption of small strains and displacements (Fig. 1). The boundary $\Gamma^{\alpha}$ of $\Omega^{\alpha}(\alpha=1,2)$ is divided into three disjoint parts $\Gamma_{D}^{\alpha}, \Gamma_{N}^{\alpha}$ and $\Gamma_{C}^{\alpha}$ with $\Gamma_{D}^{\alpha} \neq \emptyset$. On the first part denoted by $\Gamma_{D}^{\alpha}$ we assume homogeneous Dirichlet data. On the second part denoted by $\Gamma_{N}^{\alpha}$ a surface density force $p^{\alpha}$ is given. The complementary part denoted by $\Gamma_{C}^{\alpha}$ is the boundary region where the contact between the two bodies is possible. We suppose that $\Gamma_{\mathrm{C}}^{1}=\Gamma_{\mathrm{C}}^{2}$, 


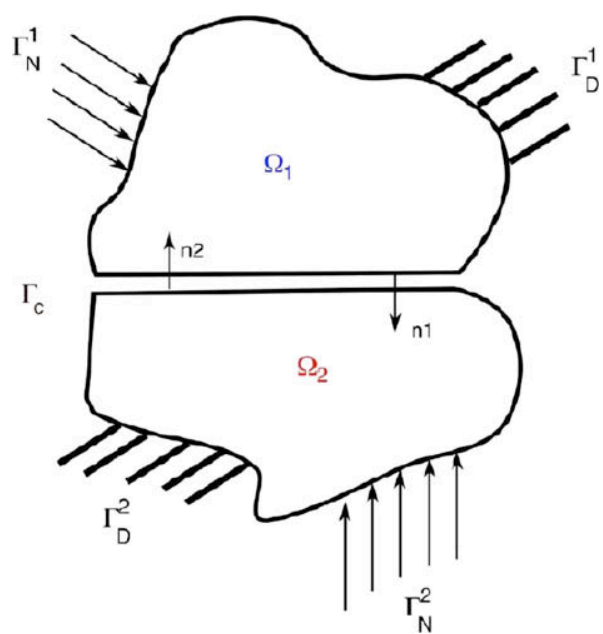

Fig. 1. Contact problem.

which we denote $\Gamma_{\mathrm{C}}$. The notation $n^{\alpha}$ stands for the unit outward normal on the boundary of $\Omega^{\alpha}$. We choose an orientation for $\Gamma_{C}$ by setting: $n^{c}=n^{1}$. Furthermore, each body is subjected to a volume force $b^{\alpha}$. We assume that the strain tensor $\varepsilon$ generated by the displacement field $u$ is linearized, and we denote by $\mathbf{K}^{\alpha}$ the elastic operator associated with $\Omega^{\alpha}$.

\subsection{Problem formulation}

The problem of unilateral contact consists of finding $\left(u^{\alpha}, \sigma^{\alpha}\right)$ defined on $\Omega^{\alpha}(\alpha=1,2)$ satisfying conditions (1)-(6)

$u^{\alpha}=0 \quad$ on $\Gamma_{D}^{\alpha}$,

$\operatorname{div} \sigma^{\alpha}+b^{\alpha}=0$ in $\Omega^{\alpha}$,

$\sigma^{\alpha} n^{\alpha}=p^{\alpha} \quad$ on $\Gamma_{N}^{\alpha}$,

$\sigma^{\alpha}=\mathbf{K}^{\alpha} \varepsilon\left(u^{\alpha}\right)$ in $\Omega^{\alpha}$.

The unilateral contact relations are satisfied on $\Gamma_{\mathrm{C}}$

$t_{N}\left(\sigma^{1}\right)=-t_{N}\left(\sigma^{2}\right)=t_{N}^{c}, \quad t_{T}\left(\sigma^{1}\right)=t_{T}\left(\sigma^{2}\right)=0$,

$\left(u_{N}^{1}-u_{N}^{2}\right) \leqslant 0, \quad t_{N}^{c} \leqslant 0, \quad\left(u_{N}^{1}-u_{N}^{2}\right) t_{N}^{c}=0$,

where

$t\left(\sigma^{\alpha}\right)=\sigma^{\alpha} n^{\alpha}$,

and for any vector $x$ we define its normal and tangential components by

$x_{N}=x \cdot n^{c}, \quad x_{T}=x-x_{N} n^{c}$.

The equalities in Eq. (5) express the continuity of the normal stress between the two elastic bodies, and the absence of friction. The first inequality in Eq. (6) expresses the non-penetration of the two bodies: either contact or separation is allowed. The second inequality states the sign of the normal constraint. The third condition implies that at the possible contact area $\Gamma_{\mathrm{C}}$, we have either zero boundary stress or contact between the two bodies.

\section{Domain decomposition algorithm}

In this section, we briefly recall the Neumann-Dirichlet domain decomposition algorithm that we use to solve the unilateral contact problem. According to [3], the basic idea of this algorithm is to retain the natural interface between the two bodies as numerical interface for the domain decomposition.

We define the following algorithm, in which $\theta_{p}$ is a non-negative parameter that will be determined in order to ensure the con- vergence of the algorithm. Let $t_{0}^{\text {neu }}$, a traction distribution defined on $\Gamma_{C}$, for $p \geqslant 1$, we built the sequence of functions $\left(u_{p}^{1}\right)_{p \geqslant 1}$, $\left(u_{p}^{2}\right)_{p \geqslant 1}$ and $\left(t_{p}^{\text {neu }}\right)_{p \geqslant 1}$ by solving successively, a Neumann problem $\mathcal{P}_{N}$ defined on $\Omega^{2}$, a Dirichlet problem $\mathcal{P}_{D}$ defined on $\Omega^{1}$, and by updating the traction $t_{p}^{\text {neu }}$ on $\Gamma_{C}$ :

- $\left(\mathcal{P}_{N}\right)$ For a given $t_{p-1}^{\text {neu }}$ on $\Gamma_{C}$, find a solution $\left(u_{p}^{2}, \sigma_{p}^{2}\right)$ such that

$$
\begin{aligned}
& u_{p}^{2}=0 \quad \text { on } \Gamma_{D}^{2}, \\
& \operatorname{div} \sigma_{p}^{2}+b^{2}=0 \text { in } \Omega^{2}, \\
& \sigma_{p}^{2} n^{2}=p^{2} \text { on } \Gamma_{N}^{2}, \\
& \sigma_{p}^{2} n^{2}=t_{p-1}^{n e u} \text { on } \Gamma_{C}, \\
& \sigma_{p}^{2}=\mathbf{K}^{2} \varepsilon\left(u_{p}^{2}\right) \text { in } \Omega^{2} .
\end{aligned}
$$

- $\left(\mathcal{P}_{D}\right)$ For a given $u_{p}^{2}$ on $\Gamma_{C}$, find a solution $\left(u_{p}^{1}, \sigma_{p}^{1}\right)$ such that

$$
\begin{aligned}
& u_{p}^{1}=0 \quad \text { on } \Gamma_{D}^{1}, \\
& \operatorname{div} \sigma_{p}^{1}+b^{1}=0 \quad \text { in } \Omega^{1}, \\
& \sigma_{p}^{1} n^{1}=p^{1} \quad \text { on } \Gamma_{N}^{1}, \\
& \sigma_{p}^{1}=\mathbf{K}^{1} \varepsilon\left(u_{p}^{1}\right) \quad \text { in } \Omega^{1}, \\
& t_{T}\left(\sigma_{p}^{1}\right)=0 \text { on } \Gamma_{C}, \\
& \left(\left(u_{p}^{1}\right)_{N}-\left(u_{p}^{2}\right)_{N}\right) \leqslant 0, \quad t_{N}\left(\sigma_{p}^{1}\right) \leqslant 0, \\
& \left(\left(u_{p}^{1}\right)_{N}-\left(u_{p}^{2}\right)_{N}\right) t_{N}\left(\sigma_{p}^{1}\right)=0 \quad \text { on } \Gamma_{C} .
\end{aligned}
$$

- Update $t_{p}^{\text {neu }}$

$$
t_{p}^{\text {neu }}=\theta_{p} t\left(\sigma_{p}^{1}\right)+\left(1-\theta_{p}\right) t_{p-1}^{\text {neu }}
$$

The proof of the convergence of the algorithm toward the solution of the unilateral contact problem (1)-(6) is given for $0<\theta_{p}<\theta^{*}$ in [3].

\section{Remarks}

1. In practice, we will set $\theta_{p}$ to a fixed value $\theta$. On the studied numerical examples, a choice of $\theta=0.5$ leads to the convergence of the algorithm.

2. This algorithm assumes that $\Gamma_{D}^{2} \neq \emptyset$, so the Neumann problem has a solution for every $t_{p}^{\text {neu }}$.

\section{Variational and discrete formulation of the algorithm}

\subsection{Variational formulation}

In order to obtain the variational formulation of the problems, we introduce the spaces $\mathcal{V}^{\alpha}(\alpha=1,2)$

$\mathcal{V}^{\alpha}=\left\{v \in\left(H^{1}\left(\Omega^{\alpha}\right)\right)^{2}, v=0\right.$ on $\left.\Gamma_{D}^{\alpha}\right\}$

and $\mathcal{N}\left(\Gamma_{\mathrm{C}}\right)$ the convex cone of negative functions defined on $\Gamma_{\mathrm{C}}$ in some dual sense (for a detailed study see for instance [15]).

For $v \in \mathcal{V}^{\alpha}$ and $u \in \mathcal{V}^{\alpha}$, we set

$a_{\alpha}(u, v)=\int_{\Omega^{\alpha}} \mathbf{K}^{\alpha} \varepsilon\left(u^{\alpha}\right) \cdot \varepsilon\left(v^{\alpha}\right) d \Omega$

and

$f_{\alpha}(v)=\left(\int_{\Omega^{\alpha}} b^{\alpha} \cdot v d \Omega+\int_{\Gamma_{N}^{\alpha}} p^{\alpha} \cdot v d S\right)$,

where $a_{\alpha}(\cdot, \cdot)$ is the bilinear form in elasticity. The linear form $f_{\alpha}(\cdot)$ takes into account the external loads $b^{\alpha}$ and $p^{\alpha}$. 


\subsubsection{Neumann problem $\mathcal{P}_{N}$}

The problem $\mathcal{P}_{N}$ is a classical linear elastic problem with a prescribed traction on $\Gamma_{C}$. The weak form of Eqs. (7)-(11) is given by a variational equality

Find $u_{p}^{2} \in \mathcal{V}^{2}$ such that $a_{2}\left(u_{p}^{2}, v\right)$

$$
=f_{2}(v)+\int_{\Gamma_{C}} t_{p-1}^{\text {neu }} \cdot v d S \quad \forall v \in \mathcal{V}^{2} .
$$

\subsubsection{Dirichlet problem $\mathcal{P}_{D}$}

The problem $\mathcal{P}_{D}$ is a unilateral contact problem on a rigid body. The convex set of admissible displacement that contains the displacement fields satisfying the non-penetration condition is defined by

$\mathcal{V}_{a d}^{1}=\left\{v \in \mathcal{V}^{1}, v \cdot n^{c}-\delta_{p} \leqslant 0\right.$ on $\left.\Gamma_{C}\right\}$,

where $\delta_{p}=u_{p}^{2} \cdot n^{c}$ describes the rigid body.

It is well known that the weak form solution of the unilateral contact problem defined by Eqs. (12)-(17) can be obtained from a variational inequality

Find $u_{p}^{1} \in \mathcal{V}_{a d}^{1}$ such that $a_{1}\left(u_{p}^{1}, v-u_{p}^{1}\right) \geqslant f_{1}\left(v-u_{p}^{1}\right) \quad \forall v \in \mathcal{V}_{a d}^{1}$

Introducing the surface traction on the contact boundary $\Gamma_{C}$ as additional unknown, the variational inequality (20) can be written as a saddle point problem: Find $\left(u_{p}^{1}, \lambda\right) \in \mathcal{V}^{1} \times \mathcal{N}\left(\Gamma_{C}\right)$ such that

$a_{1}\left(u_{p}^{1}, v\right)-f_{1}(v)-\int_{\Gamma_{C}} \lambda\left(v \cdot n^{c}\right) d S=0 \quad \forall v \in \mathcal{V}^{1}$,
$\int_{\Gamma_{C}}(\mu-\lambda)\left(u_{p}^{1} \cdot n^{c}-\delta_{p}\right) d S \geqslant 0 \quad \forall \mu \in \mathcal{N}\left(\Gamma_{C}\right)$.

\subsection{Discretized algorithm}

With each subdomain $\Omega^{\alpha}$, we associate a regular family of discretization $T_{h \alpha}$. Here we use, classical six-node triangular elements. These triangulations coincide on the contact zone $\Gamma_{c}$. The interface $\Gamma_{C}$ is then associated with one-dimensional triangulation inherited from $T_{h 1}$ or $T_{h 2}$. We will denote the FE discretization of the space $\mathcal{V}^{\alpha}$ by $\mathcal{V}_{h}^{\alpha}$ and of the space $\mathcal{N}\left(\Gamma_{C}\right)$ by $\mathcal{N}_{h}\left(\Gamma_{C}\right)$.

\subsubsection{Discretized Neumann problem $\mathcal{P}_{N, h}$}

The finite element problem $\mathcal{P}_{N, h}$ for $\mathcal{P}_{N}$ is

Find $u_{h, p}^{2} \in \mathcal{V}_{h}^{2}$ such that $a_{2}\left(u_{h, p}^{2}, v_{h}\right)$

$$
=f_{2}\left(v_{h}\right)+\int_{\Gamma_{C}} t_{p}^{\text {neu }} \cdot v_{h} d S \quad \forall v_{h} \in \mathcal{V}_{h}^{2} .
$$

\subsubsection{Discretized Dirichlet problem $\mathcal{P}_{D, h}$}

The discretized mixed variational formulation is: Find $u_{h, p}^{1} \in \mathcal{V}_{h}^{1}$ and $\lambda_{h} \in \mathcal{N}_{h}\left(\Gamma_{\mathrm{C}}\right)$ such that

$$
\begin{aligned}
& a_{1}\left(u_{h, p}^{1}, v_{h}\right)-\int_{\Gamma_{C}} \lambda_{h}\left(v_{h} \cdot n^{c}\right) d S=0 \quad \forall v_{h} \in \mathcal{V}_{h}, \\
& \int_{\Gamma_{C}}\left(\mu_{h}-\lambda_{h, p}\right)\left(u_{h, p}^{1} \cdot n^{c}-\delta_{h, p}\right) d S \geqslant 0 \quad \forall \mu_{h} \in \mathcal{N}_{h}\left(\Gamma_{C}\right),
\end{aligned}
$$

where $\delta_{h, p}=u_{h, p}^{2} \cdot n^{c}$, describes the shape of the rigid obstacle.

4.2.3. Matrix formulation of the Neumann-Dirichlet algorithm

To build the matrix formulation of the discretized problems (22) and (23), we need to introduce the expressions of the functions $u_{h}^{\alpha}, \lambda_{h}$ by considering their values at the $i$ th node of the FE discretization
$u_{h}^{\alpha}=\sum_{i=1}^{N_{\alpha}} \phi_{i}^{\alpha} q^{\alpha}(i), \quad \lambda_{h}=\sum_{i=1}^{N_{c}} \psi_{i} \Lambda(i)$,

where $\phi_{i}^{\alpha}, \psi_{i}$ are the basis functions of the spaces $\mathcal{V}_{h}^{\alpha}, \mathcal{N}_{h}\left(\Gamma_{C}\right)$ (i.e. $\phi_{i}^{\alpha}$ are the basis functions for a six-node triangular element, and $\psi_{i}$ their restriction to $\Gamma_{C}$ ).

The normal part of $t_{p}^{\text {neu }}$ can be expressed on that basis (the tangential part is set to zero as there is no friction)

$t_{p}^{\text {neu }} \cdot n^{c}=\sum_{i=1}^{N_{c}} \psi_{i} \Lambda_{p}^{\text {neu }}(i)$

The FE discretization of the domain decomposition algorithm (12)-(18) leads to the following discretized algorithm: Let $\Lambda_{0}^{\text {neu }} \in \mathbb{R}^{N_{c}}$ be given, at each iteration $p$ solve the following problems:

- For a given $\Lambda_{p-1}^{n e u}$, compute a solution $q_{p}^{2}$ of the discretized Neumann problem

$$
\mathbf{K}^{2} q_{p}^{2}=F^{2}+\left(\begin{array}{l}
\mathbf{C} \\
0
\end{array}\right) \Lambda_{p-1}^{\text {neu }} .
$$

- For a given $q_{p}^{2}$, compute a solution $\left(q_{p}^{1}, \Lambda_{p}^{\text {dir }}\right)$ of the discretized Dirichlet problem

$$
\begin{aligned}
& \mathbf{K}^{1} q_{p}^{1}-\left(\begin{array}{c}
\mathbf{C} \\
0
\end{array}\right) \Lambda_{p}^{\text {dir }}=F^{1}, \\
& \left(q_{p, N}^{1}-q_{p, N}^{2}\right) \leqslant 0, \quad \mathbf{C} \Lambda_{p}^{\text {dir }} \leqslant 0, \quad\left(q_{p, N}^{1}-q_{p, N}^{2}\right)^{T} \mathbf{C} \Lambda_{p}^{d i r}=0,
\end{aligned}
$$

where $q_{p, N}^{\alpha}$ is the vector corresponding to the normal displacements of the nodes of $\Omega^{\alpha}$ on $\Gamma_{C}$. $\mathbf{K}^{\alpha}(\alpha=1,2)$ is the stiffness matrix corresponding to $\Omega^{\alpha}, \mathbf{C}$ is the contact matrix and $F^{\alpha}$ is the vector representing the external loads.

- Update $\Lambda_{p}^{\text {neu }}$

$$
\Lambda_{p}^{\text {neu }}=\theta_{p} \Lambda_{p}^{\text {dir }}+\left(1-\theta_{p}\right) \Lambda_{p-1}^{\text {neu }} .
$$

The stopping criterion is defined by

$\varepsilon_{\tau}=\frac{\left\|\Lambda_{p}^{\text {dir }}-\Lambda_{p-1}^{\text {neu }}\right\|}{1 / 2\left\|\Lambda_{p}^{\text {dir }}+\Lambda_{p-1}^{\text {neu }}\right\|}$

At each step of the algorithm the elastic linear problem (25) is solved for a given traction on the contact zone. The problem (26) is a unilateral contact problem on a rigid body which is solved by a status method as defined in the finite element code CASTEM2000 [16]. On the studied problems, the status method converges in 2 or 3 iterations toward a solution verifying

$q_{p, N}^{1}-q_{p, N}^{2} \leqslant 0$ and $\mathbf{C} \Lambda_{p}^{\text {neu }} \leqslant 0$.

When converged this algorithm gives us two displacements vectors $\left(q^{1}, q^{2}\right)$ and two contact forces vectors $\left(\Lambda^{1}=\Lambda^{\text {dir }}\right.$, $\left.\Lambda^{2}=\Lambda^{\text {neu }}\right)$. The solution is computed from the discrete vectors

$u_{h}^{\alpha}=\sum_{i=1}^{N_{1}} \phi_{i}^{\alpha} q^{\alpha}(i) \quad$ and $\quad \sigma_{h}^{\alpha}=\mathbf{K}^{\alpha} \varepsilon\left(u_{h}^{\alpha}\right) \quad$ on $\Omega^{\alpha}$,

$t_{h}^{\alpha}=\sum_{i=1}^{N_{c}} \psi_{i} \Lambda^{\alpha}(i) \quad$ on $\Gamma_{C}$

This algorithm introduces two error sources, the first one is introduced by the resolution of the FE problems (Eqs. (25) and (26)), the second one is introduced by the iterative NeumannDirichlet algorithm. 


\section{Error estimation}

The approach based on the constitutive relation error (CRE) relies on a partition of the equations of the reference problem to be solved into two groups [6]. In elasticity, the first group consists of the kinematic constraints and the equilibrium equations while the constitutive relation forms the second group. The quality of an approximate solution satisfying the first group (i.e. an admissible solution) is quantified by the non-fulfilment of the second group of equations (i.e. the constitutive relation).

\subsection{Contact modelling and problem formulation}

In order to clearly express the error in the constitutive relation, we follow the presentation proposed in [9] and we consider the interface $\Gamma_{C}$ as a mechanical entity which has its own constitutive relation. We introduce on the interface $\Gamma_{C}$ the functions $w^{1}, w^{2}$, representing two displacement fields (one on each side of the interface), $t^{1}, t^{2}$, representing two fields of surface density forces (stresses transmitted to $\Omega^{1}$ and $\Omega^{2}$ ) and $t^{c}$ an "interior" field of surface density forces.

The kinematic conditions on the interface are expressed by

$w^{1}=u^{1} \quad$ and $\quad w^{2}=u^{2} \quad$ on $\Gamma_{\mathrm{C}}$.

The equilibrium of the interface is expressed by

$t^{c}=t^{1}$ and $t^{c}=-t^{2}$ on $\Gamma_{C}$.

Let us define the jump of displacement $w^{c}$ which, for the interface, plays a similar role as a strain

$w^{c}=w^{1}-w^{2} \quad$ on $\Gamma_{C}$.

Coulomb's constitutive law in a frictionless case, can be formulated as follows:

$w_{N}^{c} \leqslant 0, \quad t_{N}^{c} \leqslant 0, \quad t_{N}^{c} w_{N}^{c}=0 \quad$ and $\quad t_{T}^{c}=0 \quad$ on $\Gamma_{C}$,

Following $[17,18]$, the Coulomb's constitutive law in a frictionless case defined by Eq. (31) is equivalent to the condition

$\phi\left(-w^{c}\right)+\phi^{*}\left(t^{c}\right)+t^{c} \cdot w^{c}=0$ on $\Gamma_{C}$,

where the convex potentials $\phi$ and $\phi^{*}$ are defined by

$\phi(v)=\left\{\begin{array}{ll}0 & \text { if } v_{N} \geqslant 0, \\ +\infty & \text { otherwise, }\end{array}\right.$ and

$\phi^{*}(g)= \begin{cases}0 & \text { if } g_{N} \leqslant 0 \text { and } g_{T}=0, \\ +\infty & \text { otherwise, }\end{cases}$

moreover for any pair $(w, t)$ defined on $\Gamma_{c}$, the Legendre-Fenchel inequality leads to

$\phi(-w)+\phi^{*}(t)+t \cdot w \geqslant 0$.

The problem of unilateral contact defined by conditions (1)-(6) is formulated as follows: Find $\left(u^{\alpha}, \sigma^{\alpha}\right)$ defined on $\Omega^{\alpha}(\alpha=1,2)$ and $\left(w^{1}, w^{2}, t^{1}, t^{2}, t^{c}\right)$ defined on $\Gamma_{C}$ such that

- $\left(u^{\alpha}, w^{\alpha}\right)$ satisfy the kinematic conditions $u^{\alpha}=0 \quad$ on $\Gamma_{D}^{\alpha} \quad$ and $\quad u^{\alpha}-w^{\alpha}=0$ on $\Gamma_{C}$.

- $\left(\sigma^{\alpha}, t^{\alpha}, t^{c}\right)$ satisfy the equilibrium equations

$\operatorname{div} \sigma^{\alpha}+b^{\alpha}=0$ in $\Omega^{\alpha}$,

$\sigma^{\alpha} n^{\alpha}=p^{\alpha} \quad$ on $\Gamma_{N}^{\alpha}$

$\sigma^{\alpha} n^{\alpha}=t^{\alpha} \quad$ on $\Gamma_{C}$,

$t^{c}-t^{1}=0$ and $t^{c}+t^{2}=0$ on $\Gamma_{C}$.

- $\left(u^{\alpha}, \sigma^{\alpha}\right)$ satisfy the elastic constitutive relation

$\sigma^{\alpha}=\mathbf{K}^{\alpha} \varepsilon\left(u^{\alpha}\right)$ in $\Omega^{\alpha}$.
- $\left(w^{c}=w^{1}-w^{2}, t^{c}\right)$ satisfy the contact constitutive relation

$$
\phi\left(-w^{c}\right)+\phi^{*}\left(t^{c}\right)+t^{c} \cdot w^{c}=0 \quad \text { on } \Gamma_{C} .
$$

The solution of the unilateral contact problem is denoted $s=(u, c)$ with $u=\left(u^{1}, u^{2}, w^{1}, w^{2}\right)$ and $c=\left(\sigma^{1}, \sigma^{2}, t^{1}, t^{2}, t^{c}\right)$.

\subsection{Definition of the global error estimator}

The reference problem is defined by equations (35)-(38). Let us consider an approximate solution of the problem, denoted by $\hat{s}=(\hat{u}, \hat{c}), \hat{u}=\left(\hat{u}^{1}, \hat{u}^{2}, \hat{w}^{1}, \hat{w}^{2}\right)$ and $\hat{c}=\left(\hat{\sigma}^{1}, \hat{\sigma}^{2}, \hat{t}^{1}, \hat{t}^{2}, \hat{t}^{c}\right)$ that satisfies the first group of equations:

- The fields $\left(\hat{u}^{1}, \hat{u}^{2}, \hat{w}^{1}, \hat{w}^{2}\right)$ satisfy Eq. (35).

- The fields $\left(\hat{\sigma}^{1}, \hat{\sigma}^{2}, \hat{t}^{1}, \hat{t}^{2}, \hat{t}^{c}\right)$ satisfy Eq. (36).

The solution $\hat{s}=(\hat{u}, \hat{c})$ is then said to be an admissible solution. If $\hat{s}$ satisfies the constitutive relations Eqs. (37), (38) in $\Omega$, then $\hat{s}=s$ (i.e. the exact solution is found). However, if $(\hat{u}, \hat{c})$ does not satisfy the constitutive relation, the quality of this admissible solution is measured by the CRE estimator $e_{\mathrm{CRE}}(\hat{s})$

$e_{\mathrm{CRE}}(\hat{S})=\left[\sum_{\alpha=1}^{2}\left\|\hat{\sigma}^{\alpha}-\mathbf{K}^{\alpha} \varepsilon\left(\hat{u}^{\alpha}\right)\right\|_{\sigma, \Omega^{\alpha}}^{2}+2 \int_{\Gamma_{C}}\left(\phi\left(-\hat{w}^{c}\right)+\phi^{*}\left(\hat{t}^{c}\right)+\hat{t}^{c} \hat{w}^{c}\right) d S\right]^{1 / 2}$

with $\hat{w}^{c}=\hat{w}^{1}-\hat{w}^{2}$ and $\|\sigma\|_{\sigma, \Omega^{\alpha}}=\int_{\Omega^{\alpha}}\left(\mathbf{K}^{\alpha}\right)^{-1} \sigma \cdot \sigma d \Omega$.

$e_{\mathrm{CRE}}(\hat{S})$ is the constitutive relation error estimator for the admissible solution $\hat{s}$. This CRE estimator is equal to zero if and only if the solution $\hat{s}$ is the exact solution $s$ of the unilateral contact problem. It takes into account the accuracy of both the finite element discretization and the iterative resolution of the algorithm. In [9] the authors show that the Prager-Synge's theorem in elasticity [19] can be extended to the more general unilateral contact case and that this error estimator is an upper bound of a solution error, i.e.

$e_{\mathrm{CRE}}(\hat{s}) \geqslant \sqrt{\sum_{\alpha=1}^{2}\left(\left\|u^{\alpha}-\hat{u}^{\alpha}\right\|_{u, \Omega^{\alpha}}^{2}+\left\|\hat{\sigma}^{\alpha}-\sigma^{\alpha}\right\|_{\sigma, \Omega^{\alpha}}^{2}\right)}$

with $\|u\|_{u, \Omega^{\alpha}}=\int_{\Omega^{\alpha}} \mathbf{K}^{\alpha} \varepsilon(u) \cdot \varepsilon(u) d \Omega$.

\subsection{Computation of the global error estimator}

The first step for the computation of the error estimator is to recover admissible fields from the FE solution. We need to build an admissible solution $\hat{s}_{h}=\left(\hat{u}_{h}, \hat{c}_{h}\right)$ such that

- the fields $\hat{u}_{h}=\left(\hat{u}_{h}^{1}, \hat{u}_{h}^{2}, \hat{w}_{h}^{1}, \hat{w}_{h}^{2}\right)$ satisfy Eq. (35),

- the fields $\hat{c}_{h}=\left(\hat{\sigma}_{h}^{1}, \hat{\sigma}_{h}^{2}, \hat{t}_{h}^{1}, \hat{t}_{h}^{2}, \hat{t}_{h}^{c}\right)$ satisfy Eq. (36),

- moreover, the admissible fields must satisfy $\phi\left(-\hat{w}_{h}^{c}\right)=0$ and $\phi^{*}\left(\hat{t}_{h}^{c}\right)=0$ on $\Gamma_{C}$ to obtain a finite error estimator.

The admissible displacements fields are easily built, since the finite element fields satisfy the kinematic constraints

$\hat{u}_{h}^{\alpha}=u_{h}^{\alpha} \quad$ and $\quad \hat{w}_{h}^{\alpha}=w_{h}^{\alpha} \quad$ in $\Omega^{\alpha}$.

However the tractions forces computed by the algorithm do not satisfy the equilibrium conditions on the interface (36) (i.e. $t_{h}^{1}=-t_{h}^{2}$ ) nor the condition $\phi^{*}\left(\hat{t}_{h}^{c}\right)=0$ (i.e. $t_{h N}^{1} \leqslant 0$ and $-t_{h N}^{2} \leqslant 0$ on $\left.\Gamma_{C}\right)$. Hence, we propose to built the traction $\hat{t}_{h}^{c}$ in two steps:

- In a first step, a traction $\tilde{t}_{h}^{c}=\left(t_{h}^{1}-t_{h}^{2}\right) / 2$ is computed on $\Gamma_{\mathrm{c}}$.

- In a second step $\hat{t}_{h}^{c}$ is computed by solving the following minimization problem: Find $\hat{t}_{h}^{c}=\sum_{i=1}^{N_{c}} \psi_{i} \hat{\Lambda}^{c}(i)$ such that 


$$
\hat{t}_{h, N}^{c} \leqslant 0, \quad \hat{t}_{h, T}^{c}=0 \quad \text { on } \Gamma_{C}, \text { and } \hat{t}_{h}^{c} \text { minimize } \int_{\Gamma_{C}}\left(\hat{t}_{h}^{c}-\tilde{t}_{h}^{c}\right)^{2} d \Gamma
$$

The values of $\hat{t}_{h}^{1}$ and $\hat{t}_{h}^{2}$ are then easily computed by setting $\hat{t}_{h}^{1}=\hat{t}_{h}^{c}$ and $\hat{t}_{h}^{2}=-\hat{t}_{h}^{c}$.

Remark. As specified in Section 3, we assume $\Gamma_{D}^{2} \neq \emptyset$. However, if $\Gamma_{D}^{1}=\emptyset$ a supplementary condition arises: $\hat{t}_{h}^{c}$ must satisfy the global equilibrium for each rigid body motion $u_{r b}^{1}$ defined on $\Omega^{1}$. In that case we must add this constraint in the minimization problem defined in the second step.

To build the admissible stress fields $\left(\hat{\sigma}_{h}^{1}, \hat{\sigma}_{h}^{2}\right)$ we use the techniques developed in [20]. These techniques allow to recover an equilibrated stress field from a stress field that satisfy the FE equilibrium. As the FE stress fields $\sigma_{h}^{\alpha}=\mathbf{K}^{\alpha} \varepsilon\left(u_{h}^{\alpha}\right)$ are equilibrated in the FE element sense with $t_{h}^{\alpha}$ but not with $\hat{t}_{h}^{\alpha}$, we compute two intermediate stress fields $\tilde{\sigma}_{h}^{\alpha}=\mathbf{K}^{\alpha} \varepsilon\left(\tilde{u}_{h}^{\alpha}\right)$ on each subdomain $\Omega^{\alpha}(\alpha=1,2)$ such that $\tilde{u}_{h}^{\alpha} \in \mathcal{V}_{h}^{\alpha}$ and

$$
\begin{aligned}
\int_{\Omega^{\alpha}} \mathbf{K}^{\alpha} \varepsilon\left(\tilde{u}_{h}^{\alpha}\right) \varepsilon(v) d \Omega= & \int_{\Omega^{\alpha}} b^{\alpha} \cdot v d \Omega+\int_{\Gamma_{N}^{\alpha}} p^{\alpha} \cdot v d S \\
& +\int_{\Gamma_{\mathrm{C}}} \hat{t}_{h}^{\alpha} \cdot v d S \quad \forall v \in \mathcal{V}_{h}^{\alpha} .
\end{aligned}
$$

The recovery of the stress fields $\hat{\sigma}_{h}^{\alpha}$ on each subdomain $\Omega^{\alpha}$ starting from the stress fields $\tilde{\sigma}_{h}^{\alpha}$ can then follow the classical procedure (see, e.g., $[6,21,22,20])$. Once the fields $\hat{s}_{h}$ are computed, the error estimate $\eta_{h}^{\text {glob }}$ is readily obtained from (39) by

$\eta_{h}^{\text {glob }}=e_{\mathrm{CRE}}\left(\hat{s}_{h}\right)$.

The contribution of each element $E$ of the mesh is computed as follows:

$\eta_{h, E}^{g l o b}=\left[\left\|\hat{\sigma}_{h}^{\alpha}-\mathbf{K} \varepsilon\left(\hat{u}_{h}^{\alpha}\right)\right\|_{\sigma, E}^{2}+2 \int_{\Gamma_{C} \cap E}\left(\phi\left(-\hat{w}_{h}^{c}\right)+\phi^{*}\left(\hat{t}_{h}^{c}\right)+\hat{t}^{c} \hat{w}_{h}^{c}\right) d S\right]^{1 / 2}$,

where $\|\sigma\|_{\sigma, E}=\int_{E} \mathbf{K}^{-1} \sigma \cdot \sigma d E$.

\subsection{Error indicators definition}

In view of an efficient mesh adaptation technique, it is important to separate contributions to the global error $e_{\mathrm{CRE}}(\hat{s})$ due to the FE discretization from that due to the iterative resolution of the algorithm. The later can in fact not be controlled by a mesh adaptation technique. Following the method proposed in $[23,24]$, we propose here two simple error indicators that allow us to estimate separately the part of the error due to the FE discretization and that due to the Neumann-Dirichlet iterative algorithm. The discretization error is defined as the limit of the global error when the convergence criterion of the iterative algorithm tends to zero. The Neumann-Dirichlet iterative algorithm error is defined as the limit of the global error as the mesh size $h$ tends to zero.

\subsubsection{Discretized contact constitutive relation}

In order to express the Neumann-Dirichlet algorithm error indicator, we need to reformulate the continuous version of the contact constitutive relation Eq. (32) on the discretized problem. Let $q_{N}^{\alpha}$ denote the vector of the normal displacement of the nodes of $\Omega^{\alpha}$ on $\Gamma_{C}$ and $q_{N}^{\alpha}(i)$ its values on the ith node. From Eq. (26), the discretized contact constitutive relation on $\Gamma_{C}$ reads:

for $i=1, \ldots, N_{c}$,

$\left(q_{N}^{1}(i)-q_{N}^{2}(i)\right) \leqslant 0, \quad(\mathbf{C} \Lambda)_{i} \leqslant 0, \quad\left(q_{N}^{1}(i)-q_{N}^{2}(i)\right)(\mathbf{C} \Lambda)(i)=0$.

Then, the discretized Coulomb constitutive law defined by Eq. (43) is equivalent to for $i=1, \ldots, N_{c}$,

$\phi\left(-q_{N}^{1}(i)-q_{N}^{2}(i)\right)+\phi^{*}((\mathbf{C} \Lambda)(i))+\left(q_{N}^{1}(i)-q_{N}^{2}(i)\right)(\mathbf{C} \Lambda)(i)=0$,

where $\phi$ and $\phi^{*}$ are the convex potentials defined in Eq. (33).

\subsubsection{Neumann-Dirichlet algorithm error indicator}

The Neumann-Dirichlet algorithm error (NDA error) is estimated though a quantity called NDA error indicator. To define this error indicator, we consider a new reference problem $P_{s, h}$ obtained from the reference problem (1)-(6) by the FE spatial discretization: Find $\left(u_{s, h}^{1}, \sigma_{s, h}^{1}\right)$ defined in $\Omega^{1},\left(u_{s, h}^{2}, \sigma_{s, h}^{2}\right)$ defined in $\Omega^{2}$ and $\left(w_{s, h}^{1}, w_{s, h}^{2}, t_{s, h}^{1}, t_{s, h}^{2}, t_{s, h}^{c}\right)$ defined on $\Gamma_{C}$ such that (for $\alpha=1,2$ )

- $\left(u_{s, h}^{\alpha}, w_{s, h}^{\alpha}\right)$ satisfy the kinematic conditions

$$
u_{s, h}^{\alpha}=0 \quad \text { on } \Gamma_{D}^{\alpha}, \quad u_{s, h}^{\alpha}-w_{s, h}^{\alpha}=0 \quad \text { on } \Gamma_{C},
$$

- $\left(\sigma_{s, h}^{\alpha}, t_{s, h}^{\alpha}, t_{s, h}^{c}\right)$ satisfy the equilibrium equations of the finite element model

$$
\begin{aligned}
& t_{s, h}^{c}-t_{s, h}^{1}=0 \text { and } t_{s, h}^{c}+t_{s, h}^{2}=0 \quad \text { on } \Gamma_{C}, \\
& \int_{\Omega^{\alpha}} \sigma_{s, h}^{\alpha} \varepsilon(v) d \Omega=\int_{\Omega^{\alpha}} b^{\alpha} \cdot v d \Omega+\int_{\Gamma_{N}^{\alpha}} p^{\alpha} \cdot v d S+\int_{\Gamma_{C}} t_{s, h}^{\alpha} \cdot v d S \quad \forall v \in \mathcal{V}_{h}^{\alpha},
\end{aligned}
$$

- $\left(u_{s, h}^{\alpha}, \sigma_{s, h}^{\alpha}\right)$ satisfy the elastic constitutive relation

$$
\sigma_{s, h}^{\alpha}=\mathbf{K}^{\alpha} \varepsilon\left(u_{s, h}^{\alpha}\right) \text { in } \Omega^{\alpha},
$$

- $\left(w_{s, h}^{c}=w_{s, h}^{1}-w_{s, h}^{2}, t_{s, h}^{c}\right)$ satisfy the discretized contact constitutive relation

$$
\begin{aligned}
& \text { for } i=1, \ldots, N_{c}, \\
& \phi\left(-\left(q_{N}^{1}(i)-q_{N}^{2}(i)\right)\right)+\phi^{*}((\mathbf{C} \Lambda)(i))+\left(q_{N}^{1}(i)-q_{N}^{2}(i)\right)(\mathbf{C} \Lambda)(i)=0,
\end{aligned}
$$

where $w_{s, h}^{c}=\sum_{i=1}^{N_{c}} \phi_{i}\left(q_{N}^{1}(i)-q_{N}^{2}(i)\right)$ and $t_{s, h}^{c}=\sum_{i=1}^{N^{c}} \psi_{i} \Lambda(i)$.

The solution of the problem $P_{s, h}$ is denoted $s_{s, h}=\left(u_{s, h}, c_{s, h}\right)$ with $u_{s, h}=\left(u_{s, h}^{1}, u_{s, h}^{2}, w_{s, h}^{1}, w_{s, h}^{2}\right)$ and $c_{h}=\left(\sigma_{s, h}^{1}, \sigma_{s, h}^{2}, t_{s, h}^{1}, t_{s, h}^{2}, t_{s, h}^{c}\right)$. The only approximation introduced, between the reference solution $s_{s, h}$ and the approximate solution $s_{h}$, is the resolution of the Neumann-Dirichlet algorithm. This error can be measured by a CRE estimator computed on problem $P_{s, h}$. Hence, this CRE estimator is used as an error indicator to measure the contribution of the Neumann-Dirichlet algorithm to the global error. Let $\hat{s}_{s, h}=\left(\hat{u}_{s, h}, \hat{c}_{s, h}\right)$ be an admissible solution for the problem $P_{s, h}$ (i.e. $\hat{u}_{s, h}$ satisfies the kinematic constraints (45) and $\hat{c}_{s, h}$ satisfies the FE-equilibrium $(46,47))$. The NDA error indicator is then defined by

$$
\begin{aligned}
\eta_{h}^{\mathrm{NDA}}= & \sum_{\alpha=1}^{2}\left\|\hat{\sigma}_{s, h}^{\alpha}-\mathbf{K} \varepsilon\left(\hat{u}_{s, h}^{\alpha}\right)\right\|_{\sigma, \Omega^{\alpha}}^{2}+2 \sum_{i=1}^{N_{c}}\left(\phi\left(-\hat{q}_{N}^{c}(i)\right)+\phi^{*}\left(\left(\mathbf{C} \widehat{\Lambda}^{c}\right)(i)\right)\right. \\
& \left.+\hat{q}_{N}^{c}(i)\left(\mathbf{C} \widehat{\Lambda}^{c}\right)(i)\right)
\end{aligned}
$$

where $\hat{q}_{N}^{c}(i)$ and $\widehat{\Lambda}^{c}(i)$ are defined by $\hat{w}_{s, h}^{c}=\hat{w}_{s, h}^{1}-\hat{w}_{s, h}^{2}=\sum_{i=1}^{N_{c}} \phi_{i} \hat{q}_{N}^{c}(i)$ and $\hat{t}_{h}^{c}=\sum_{i=1}^{N_{c}} \psi_{i} \widehat{\Lambda}^{c}(i)$. This admissible solution can be easily built from $s_{h}$ by setting

$\hat{u}_{s, h}^{\alpha}=\hat{u}_{h}^{\alpha} \quad$ in $\Omega^{\alpha} \quad$ and $\quad \hat{w}_{s, h}^{\alpha}=\hat{w}_{h}^{\alpha} \quad$ on $\Gamma_{C}$,

$\hat{\sigma}_{s, h}^{\alpha}=\tilde{\sigma}_{h}^{\alpha} \quad$ in $\Omega^{\alpha} \quad$ and $\quad \hat{t}_{s, h}^{\alpha}=\hat{t}_{h}^{\alpha} \hat{t}_{s, h}^{c}=\hat{t}_{h}^{c} \quad$ on $\Gamma_{C}$,

where the three traction forces $\left(\hat{t}_{h}^{c}, \hat{t}_{h}^{1}, \hat{t}_{h}^{2}\right)$ and the stress fields $\left(\tilde{\sigma}_{h}^{1}, \tilde{\sigma}_{h}^{2}\right)$ satisfy the FE-equilibrium and have been already computed to build the global error $e_{\mathrm{CRE}}\left(s_{h}\right)$ (Section 5.3).

\subsubsection{Discretization error indicator}

The discretization error is estimated through a quantity called a discretization error indicator. To compute the discretization error 
indicator, we consider that the reference problem is the problem defined, for a given $t_{p-1}^{\text {neu }}$, by Eqs. (7)-(17). We will denote this problem by $P_{c, p}$. Let us denote by $s_{p}$ the solution of the problem $P_{c, p}$ and by $s_{h, p}$ the associated finite element solution (computed from Eqs. (25), (26)). The only approximation introduced between $s_{p}$ and $s_{h, p}$ is the FE discretization. A CRE estimator computed on problem $P_{c, p}$ can be used as an indicator to measure the contribution of the discretization error to the global error. To define a CRE estimator on $P_{c, p}$, the problem $P_{c, p}$ is written as follows: For a given $t_{p-1}^{\text {neu }}$, find $s_{p}=\left(u_{p}, c_{p}\right)$ such that

- $u_{p}=\left(u_{p}^{1}, u_{p}^{2}, w_{p}^{1}, w_{p}^{2}\right), c_{p}=\left(\sigma_{p}^{1}, \sigma_{p}^{2}, t_{p}^{1}, t_{p}^{2}, t_{p}^{c}\right)$,

- $\left(u_{p}^{\alpha}, w_{p}^{\alpha}\right)$ satisfy the kinematic conditions

$$
u_{p}^{\alpha}=0 \quad \text { on } \Gamma_{D}^{\alpha} \quad \text { and } \quad u_{p}^{\alpha}-w_{p}^{\alpha}=0 \text { on } \Gamma_{C},
$$

- $\left(\sigma_{p}^{\alpha}, t_{p}^{\alpha}, t_{p}^{c}\right)$ satisfy the equilibrium equations

$$
\begin{aligned}
& \operatorname{div} \sigma_{p}^{\alpha}+b^{\alpha}=0 \quad \text { in } \Omega^{\alpha}, \\
& \sigma_{p}^{\alpha} n_{p}^{\alpha}=p^{\alpha} \text { on } \Gamma_{N}^{\alpha}, \\
& \sigma_{p}^{\alpha} n_{p}^{\alpha}=t^{\alpha} \text { on } \Gamma_{C}, \\
& t_{p}^{c}-t_{p}^{1}=0 \text { and } t_{p-1}^{\text {neu }}+t_{p}^{2}=0 \text { on } \Gamma_{C},
\end{aligned}
$$

- $\left(u_{p}^{\alpha}, \sigma_{p}^{\alpha}\right)$ satisfy the elastic constitutive relation

$$
\sigma_{p}^{\alpha}=\mathbf{K}_{p}^{\alpha} \varepsilon\left(u_{p}^{\alpha}\right) \text { in } \Omega^{\alpha},
$$

- $\left(w_{p}^{c}=w_{p}^{1}-w_{p}^{2}, t_{p}^{c}\right)$ satisfy the contact constitutive relation

$$
\phi\left(-w_{p}^{c}\right)+\phi^{*}\left(t_{p}^{c}\right)+t_{p}^{c} w_{p}^{c}=0 \quad \text { on } \Gamma_{C} .
$$

Let $\hat{s}_{p}=\left(\hat{u}_{p}, \hat{c}_{p}\right)$ be a pair that satisfies the kinematic constraints (51) and the equilibrium equations (52) (i.e. $\hat{s}_{p}$ is an admissible solution for $P_{c, p}$ ). If $\hat{s}_{p}$ satisfies the constitutive relations (53), (54) then it is the exact solution of $P_{c, p}$. The discretization error indicator $\eta_{h, p}^{\text {dis }}$ is defined by

$\eta_{h, p}^{\text {dis }}=e_{\mathrm{CRE}}\left(\hat{\mathrm{s}}_{p}\right)$

with

$e_{\mathrm{CRE}}\left(\hat{S}_{p}\right)=\left[\sum_{\alpha=1}^{2}\left\|\hat{\sigma}_{p}^{\alpha}-\mathbf{K} \varepsilon\left(\hat{u}_{p}^{\alpha}\right)\right\|_{\sigma, \Omega^{\alpha}}^{2}+2 \int_{\Gamma_{C}}\left(\phi\left(\hat{w}_{p}^{c}\right)+\phi^{*}\left(\hat{t}_{p}^{c}\right)+\hat{t}_{p}^{c} \hat{w}_{p}^{c}\right) d S\right]^{1 / 2}$.

The reference problem $P_{c, p}$ can be split into two problems, i.e. a classical elastic problem on $\Omega^{2}$ and a unilateral contact problem on a rigid foundation on $\Omega^{1}$. Therefore, we can use the techniques developed in $[21,20]$ to build the admissible solution $\hat{s}_{p}$.

\section{Remarks}

1. The discretization error indicator $\eta_{h, p}^{\text {dis }}$ is an error estimator for the reference problem $P_{c, p}$ (i.e. it is an upper bound of the solu-

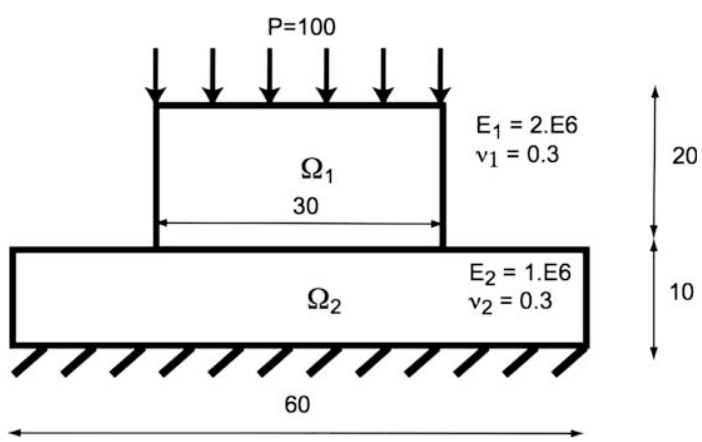

Fig. 2. Punch problem. tion error for the problem $P_{c, p}$ ), but it is only an error indicator for what we call the discretization error of the contact problem.

2. $\eta_{h, p}^{\text {dis }}$ can be decomposed into two parts. A first part defined on $\Omega^{2}$ is the classical CRE estimator in linear elasticity for a solid subjected to Dirichlet boundary conditions on $\Gamma_{D}^{2}$ and Neumann boundary conditions on $\Gamma_{N}^{2} \cap \Gamma_{\text {C }}$. A second part defined on $\Omega^{1}$ is the CRE estimator for the contact problem of an elastic body on a rigid foundation described by $w_{p}^{2}$ on $\Gamma_{c}$.

\section{Numerical results}

In this section, we study by means of two examples the behaviour of the proposed error estimator and the error indicators for a

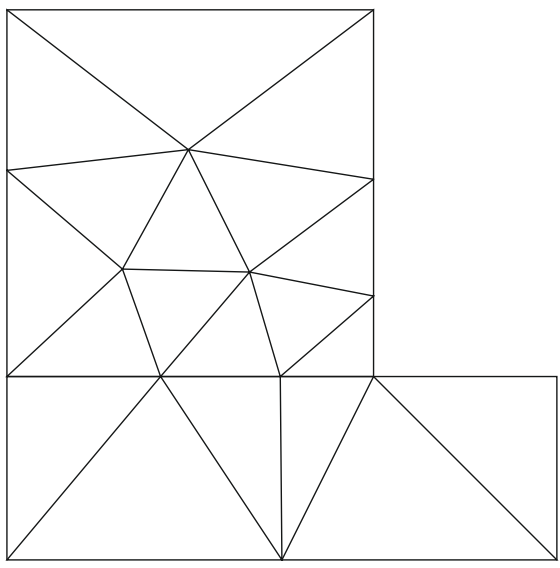

Fig. 3. Punch problem: coarse mesh.

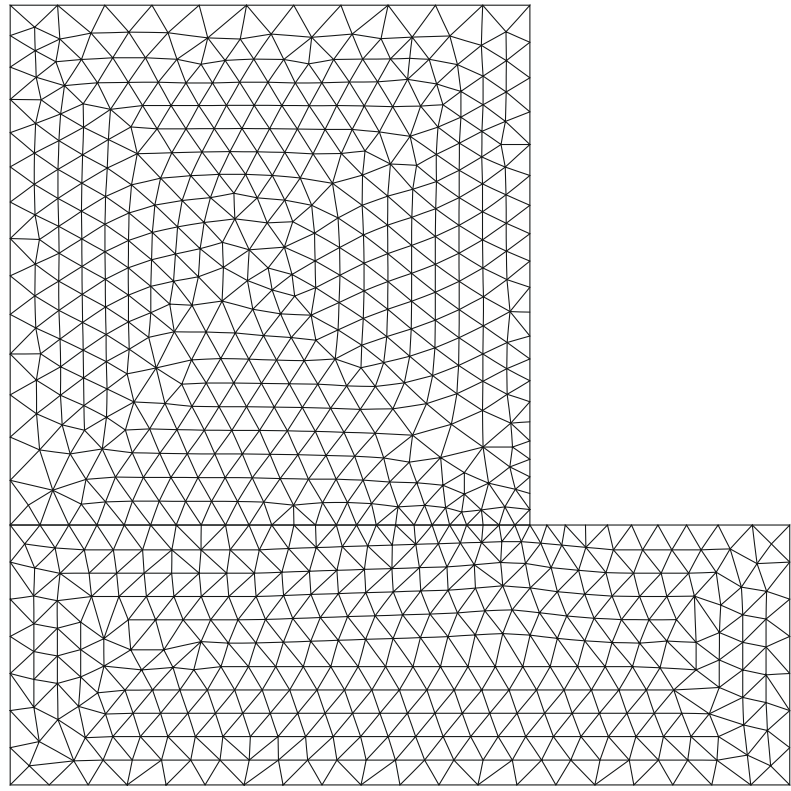

Fig. 4. Punch problem: refined mesh.

Table 1

Punch-problem: error estimator and error indicators.

\begin{tabular}{lccccccc}
\hline NDOF & 114 & 230 & 446 & 734 & 1484 & 2992 & 5864 \\
\hline$\eta_{h}^{\text {glob }} * 10^{2}$ & 10.7 & 7.59 & 6.50 & 5.52 & 4.96 & 4.61 & 4.39 \\
$\eta_{h}^{\text {dis }} * 10^{2}$ & 9.89 & 6.59 & 5.34 & 4.04 & 3.05 & 2.32 & 1.65 \\
$\eta_{h}^{\text {nda }} * 10^{2}$ & 4.02 & 4.06 & 4.06 & 4.07 & 4.07 & 4.08 & 4.08 \\
$e_{\text {ref }} * 10^{2}$ & 10.1 & 6.99 & 6.12 & 5.29 & 4.80 & 4.39 & 4.09 \\
\hline
\end{tabular}


finite element solution obtained with the Neumann-Dirichlet iterative algorithm. In general, multi-body contact problems do not admit an analytical solution. Therefore, in order to evaluate the discretization error, we compute a reference solution $u_{r e f}$ by using a finer mesh associated with a global resolution of the contact

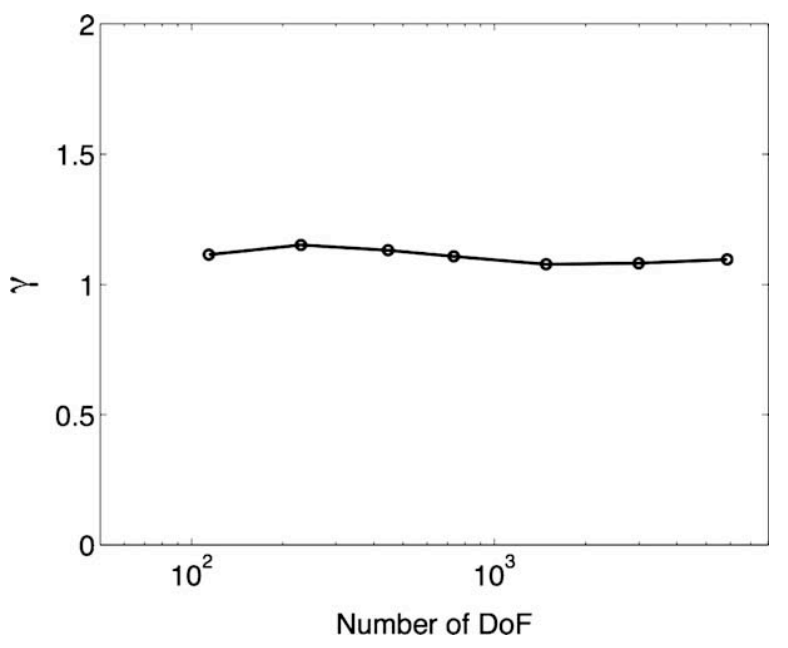

Fig. 5. Punch problem: $\gamma$ as a function of the number of degree of freedom.

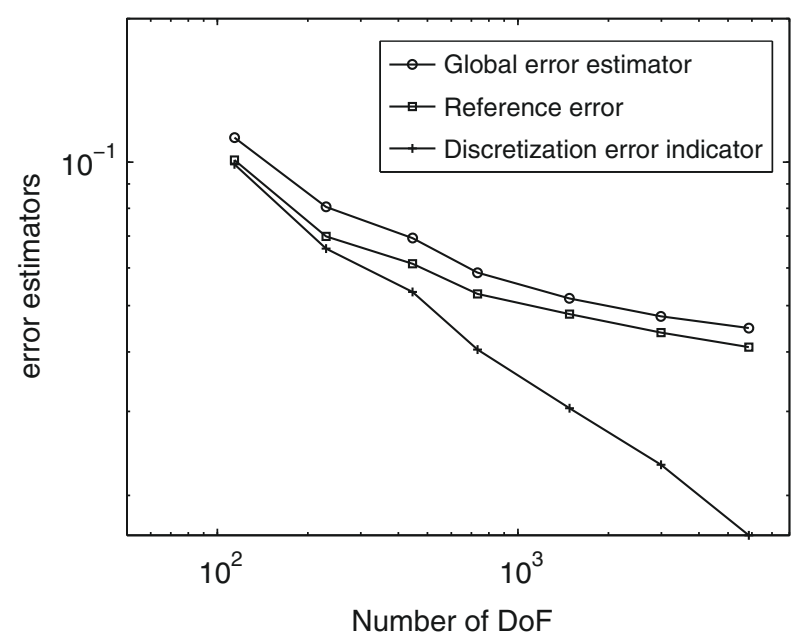

Fig. 6. Punch problem: computed errors as a function of the number of degree of freedom. problem. To obtain a reliable reference solution we choose the mesh size $h_{\text {ref }}$ equal to $\frac{1}{2^{3}} h_{\min }, h_{\min }$ being the mesh size used to compute the most refined FE solution. The reference error will be denoted $e_{\text {ref }}$ with

$e_{\text {ref }}=\sqrt{\sum_{\alpha=1}^{2}\left\|u_{h}^{\alpha}-u_{r e f}^{\alpha}\right\|_{u, \Omega^{\alpha}}^{2} .}$

\subsection{Punch problem}

In the first example we consider two elastic bodies $\Omega_{1}$ and $\Omega_{2}$ as depicted in Fig. 2 . The upper body $\Omega_{1}$ is submitted to an uniform load, and the lower body $\Omega_{2}$ is clamped. Due to the symmetry only one half of the structure is meshed. Both bodies are meshed with 6 -node triangular elements. We start with a coarse mesh (Fig. 3) and we progressively refine the mesh (Fig. 4). The coefficient $\theta$, of the Neumann-Dirichlet algorithm, is set to 0.5 and the stopping criterion $\varepsilon_{\tau}$ is chosen very large by setting it to 0.10 .

Table 1 presents the evolution of the global error estimator $\eta_{h}^{\text {glob }}$ and of the error indicators $\eta_{h}^{\text {dis }}$ and $\eta_{h}^{\mathrm{NDA}}$ as a function of the number of degrees of freedom. We have also computed $e_{r e f}$ on these meshes. Fig. 5 shows that the ratio between the global error estimator and the reference error $\gamma=\frac{\eta_{h}^{\text {glob }}}{e_{\text {ref }}}$ is very sharp and around 1.1. As expected, the global error (and the reference error) tends to an horizontal asymptote as the mesh is refined whereas the discretization error indicator has a convergence rate of about 0.9 (see Fig. 6).

The spatial distributions of the global error estimator $\eta_{h}^{\text {glob }}$ and of the discretization error indicator $\eta_{h}^{\text {dis }}$ are displayed in Fig. 7. The distribution of the values shows that the computed solution is not accurate in the structure $\Omega^{2}$. This error is mainly due to the large stopping criterion $\left(\varepsilon_{\tau}=0.10\right)$ in the iterative NeumannDirichlet algorithm, which leads to an incorrect computation of the contact forces. A second computation is performed with a smaller value for the stopping criterion $\left(\varepsilon_{\tau}=0.01\right)$. In Fig. 8 , the values of the nodal contact forces are displayed for $\varepsilon_{\tau}=0.1$, for $\varepsilon_{\tau}=0.01$ and for a reference computation performed on the same mesh with a classical global resolution of the contact problem. The nodal contact forces computed with $\varepsilon_{\tau}=0.1$ are clearly too small when compared with the reference solution whereas the values computed with $\varepsilon_{\tau}=0.01$ seem accurate. The spatial distribution of the global error estimator (Fig. 9) shows that employing a more tight tolerance in the Neuman-Dirichlet algorithm $\left(\varepsilon_{\tau}=\right.$ 0.01 ) produces much more accurate solution in $\Omega^{2}$. Moreover, note that this spatial distribution is very similar to the distribution of the discretization error displayed in Fig. 7.

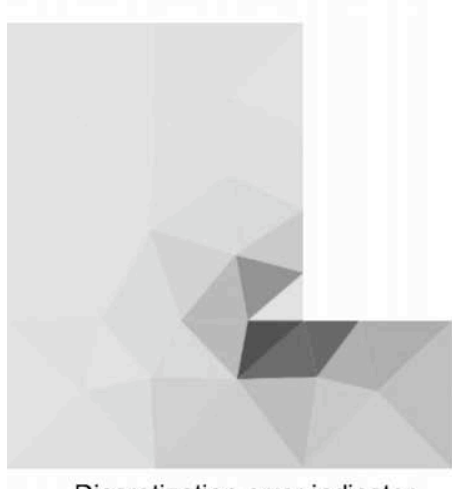

Discretization error indicator
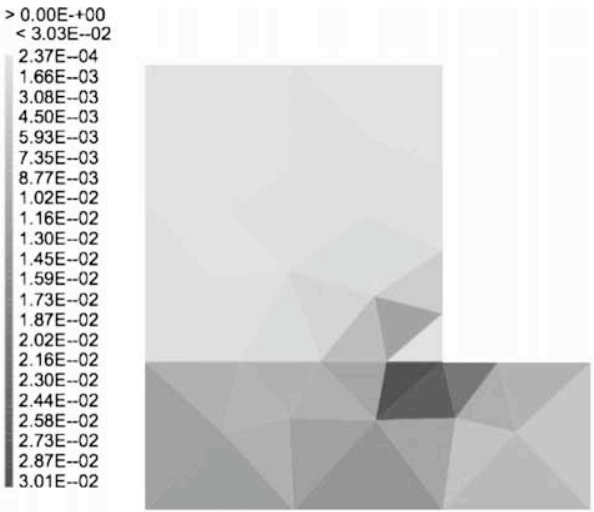

$>0.00 \mathrm{E}-+00$
$<3.45 \mathrm{E}-02$

$2.70 \mathrm{E}-04$
$1.89 \mathrm{E}-03$

$3.51 \mathrm{E}-03$

$5.13 \mathrm{E}-03$
$6.75 \mathrm{E}-03$
$0.37 \mathrm{E}-03$

$6.75 \mathrm{E}-03$
$8.37 \mathrm{E}-03$
-3.03

$9.98 \mathrm{E}-03$
$1.16 \mathrm{E}-02$

$1.16 \mathrm{E}-02$
$1.32 \mathrm{E}-02$

$1.48 \mathrm{E}-02$
$1.65 \mathrm{E}-02$

$1.65 \mathrm{E}-02$
$1.81 \mathrm{E}-02$

$1.97 \mathrm{E}-02$

2.13E-02

$2.29 \mathrm{E}-02$

$2.62 \mathrm{E}-02$

$2.78 \mathrm{E}-02$
$2.94 \mathrm{E}-02$

$3.10 \mathrm{E}-02$

3. $27 \mathrm{E}-02$

Global error estimato

Fig. 7. Punch problem: spatial error distribution. 


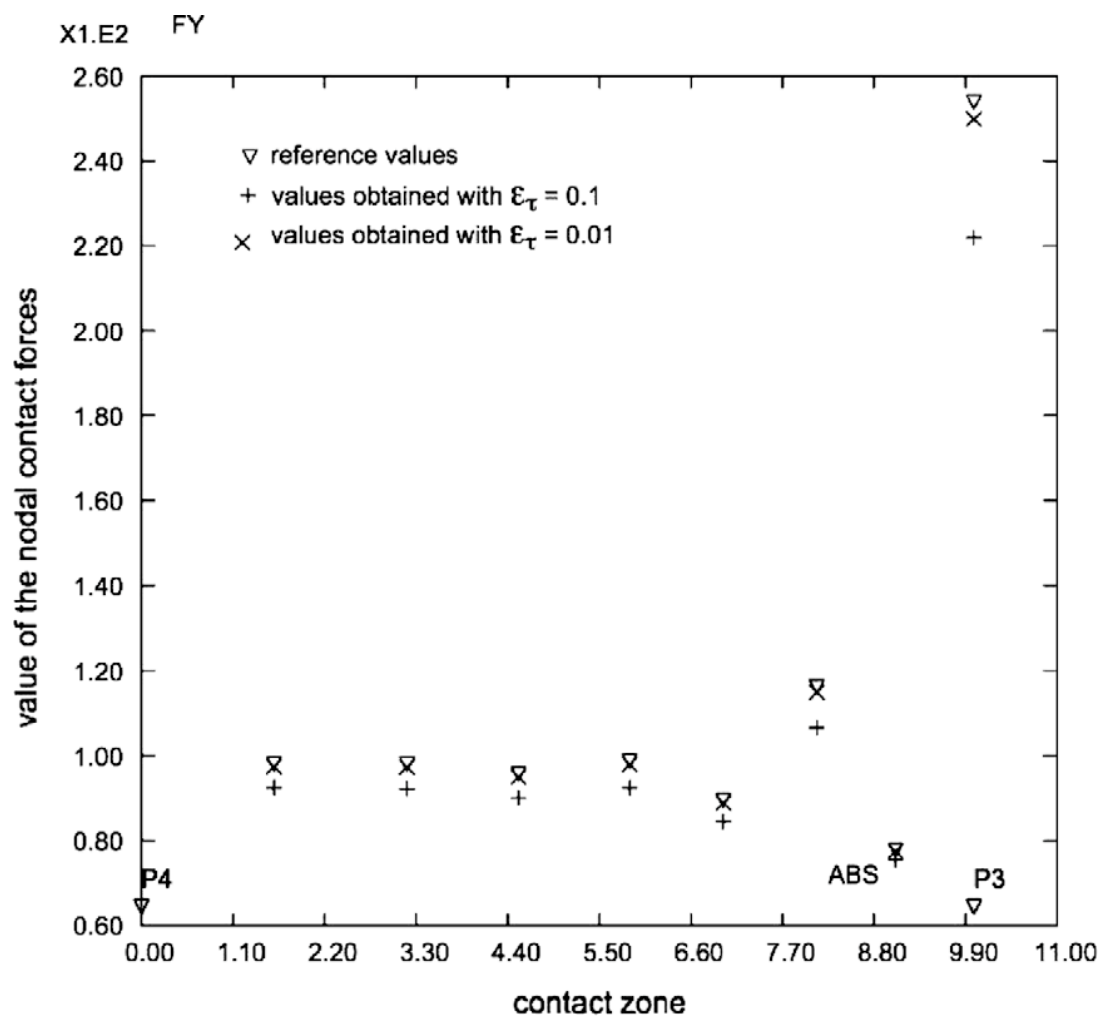

Fig. 8. Punch problem: nodal forces distribution on the contact zone.

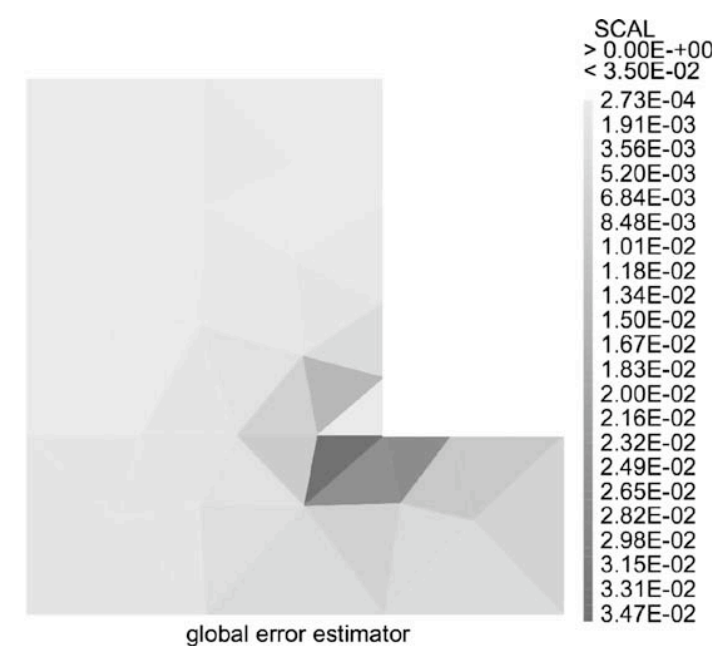

Fig. 9. Punch problem: spatial error distribution with $\varepsilon_{\tau}=0.01$.

\subsection{Punch problem with partial contact loss}

In the second example, we analyze the behaviour of the global error and of the error indicators as either the mesh or the stopping criterion is modified. The studied problem is shown in Fig. 10. The two bodies are initially in contact but the loading leads to a partial contact loss. The structure is meshed with 6-node triangular elements. In the Neumann-Dirichlet iterative algorithm, the coefficient $\theta$ is set to 0.5 .

Table 2 shows the influence of the quality of the mesh for a stopping criterion $\varepsilon_{\tau}$ set to 0.2 . The meshes 1 and 6 are shown in Figs. 11, 12. As we refine the mesh, we observe that the global error $\eta_{h}^{\text {glob }}$ initially decreases but then tends to stabilize toward what we

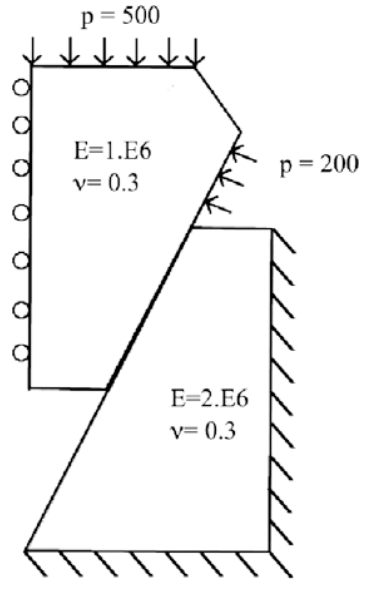

Fig. 10. Punch problem with contact loss.
Table 2

Punch-problem with contact loss: error estimator and error indicators as a function of the mesh.

\begin{tabular}{lllllll}
\hline NDOF & 712 & 1510 & 1850 & 3390 & 5534 & 6800 \\
\hline$\eta_{h}^{\text {glob }} * 10^{2}$ & 9.348 & 7.978 & 5.981 & 5.146 & 4.576 & 4.406 \\
$\eta_{h}^{\text {dis }} * 10^{2}$ & 8.442 & 6.737 & 4.222 & 2.880 & 1.706 & 1.394 \\
$\eta_{h}^{\text {nda }} * 10^{2}$ & 4.156 & 4.263 & 4.263 & 4.266 & 4.255 & 4.243 \\
\hline
\end{tabular}

have called the iteration error. The iteration error indicator $\eta_{h}^{\mathrm{NDA}}$ depends barely on the mesh discretization and approximates very well the iteration error. 


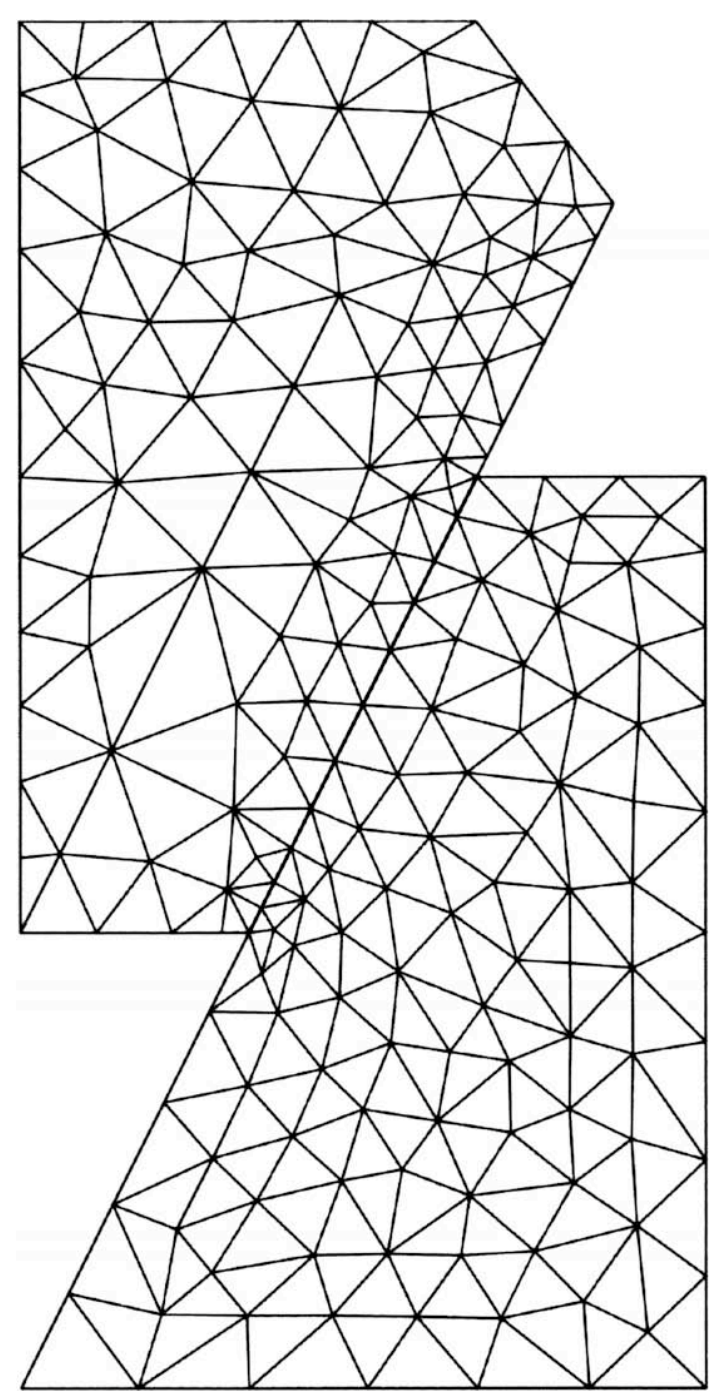

Fig. 11. Punch problem with contact loss: mesh $1-712$ DoF.

Table 3 shows the influence of the stopping criterion for a fixed mesh (see Fig. 11), $N_{n d a}$ is the number of iterations of the Neumann-Dirichlet domain decomposition algorithm. We observe that the global error $\eta_{h}^{\text {glob }}$ tends rapidly to stabilize toward what we have called the discretization error, and that the discretization error indicator $\eta_{h}^{\text {dis }}$ seems to approximate well the discretization error.

\section{Conclusion and future prospects}

This paper introduces global error estimator based on the constitutive relation to control a domain decomposition algorithm for a two-body frictionless contact problem. This error measure takes into account all the errors due to discretization, i.e. both the errors due to the spatial discretization and those due to the domain decomposition algorithm.

Two error indicators are developed to estimate the contributions of each source of error. They are defined in the same way as the error, except that the reference problem is different. On the first tests, these indicators seem to behave well.

With these tools, it would be possible to adapt the quality of the mesh during the iterations of the Neumann-Dirichlet algorithm and to control the final quality of the computation.

The next step is the generalization of Dirichlet-Neumann method to contact problems with more than two domains which

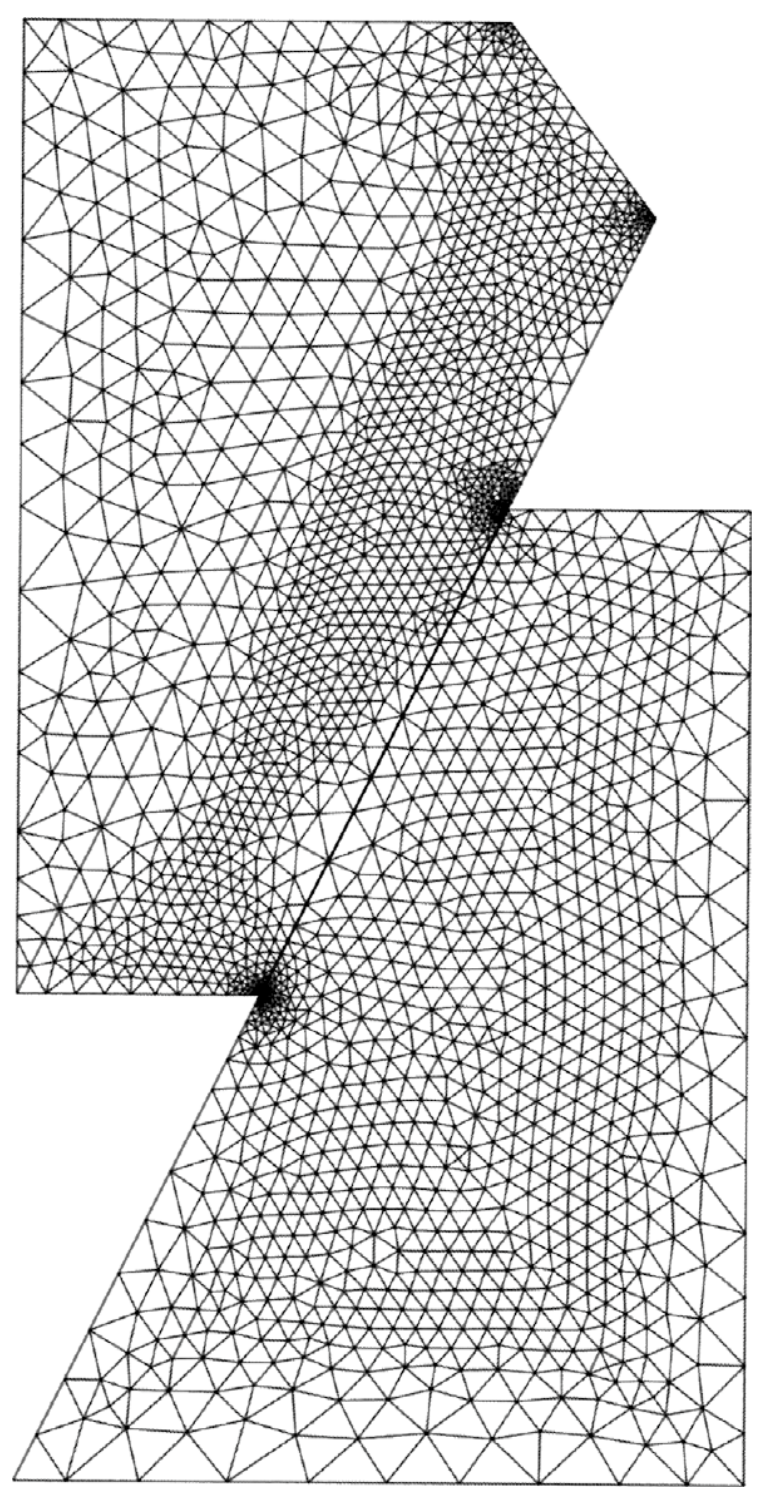

Fig. 12. Punch problem with contact loss: mesh $6-6800$ DoF.

Table 3

Punch-problem with contact loss: error estimator and error indicators as a function of the stopping criterion.

\begin{tabular}{lllllll}
\hline $\begin{array}{l}\text { Stopping } \\
\text { criteria }\end{array}$ & $2 \cdot 10^{-1}$ & $1 \cdot 10^{-1}$ & $1 \cdot 10^{-2}$ & $1 \cdot 10^{-3}$ & $1 \cdot 10^{-4}$ & $1 \cdot 10^{-8}$ \\
$N_{\text {nda }}$ & 4 & 5 & 9 & 11 & 15 & 28 \\
\hline$\eta_{h}^{\text {glob }} * 10^{2}$ & 9.348 & 8.804 & 8.657 & 8.657 & 8.657 & 8.657 \\
$\eta_{h}^{\text {dis }} * 10^{2}$ & 8.442 & 8.546 & 8.653 & 8.656 & 8.657 & 8.657 \\
$\eta_{h}^{\text {nda }} * 10^{2}$ & 4.156 & 2.279 & 0.3812 & 0.2237 & 0.0593 & 0.0066 \\
\hline
\end{tabular}

requires a specific treatment of floating subdomains by adding a global coarse problem with one or a few unknowns (rigid motions) for each subdomain [25]. It should be noted that domain decomposition algorithms for contact problem using ideas related to [25] was recently developed, for example, by Dostàl and Hòrak using scalable FETI method [26], Kronhuber and Krauss [27] gave experimental evidence of numerical scalability of algorithm based on monotone multigrid. Another approach consists to extend the balancing domain decomposition proposed in [25] by adding a coarse problem to an earlier method known as the Neumann-Neumann method [28]. This work is under consideration. 


\section{References}

[1] Kikuchi N, Oden JT, editors. Contact problems in elasticity: a study of variational inequalities and finite element methods. Philadelphia: SIAM; 1988.

[2] Wriggers P. Finite element algorithms for contact problems. Arch Comput Methods Eng 1995;2:1-49.

[3] Bayada G, Sabil J, Sassi T. Neumann-Dirichlet algorithm for unilateral contact problem: convergence results. CR Acad Sci Paris, Ser I 2002;335:381-6.

[4] Eck C, Wohlmuth B. Convergence of a contact Neumann iteration for the solution of two-body contact problems. Math Model Methods Appl Sci 2003;13(8):1103-18.

[5] Babuška I, Rheinboldt WC. A posteriori estimates for the finite element method. Int J Numer Methods Eng 1978;12:1597-615.

[6] Ladevèze P, Leguillon D. Error estimate procedure in the finite element method and application. SIAM J Numer Anal 1983;20(3):485-509.

[7] Zienkiewicz OC, Zhu JZ. A simple error estimator and adaptive procedure for practical engineering analysis. Int J Numer Methods Eng 1987;24:337-57.

[8] Wriggers P, Scherf O. Different a posteriori error estimators and indicators for contact problems. Math Comput Model 1997;28(4-8):437-47.

[9] Coorevits P, Hild P, Pelle JP. Posteriori error estimation for unilateral contact with matching and non-matching meshes. Comput Methods Appl Mech Eng 2000;186:65-83.

[10] Rieger A, Wriggers P. Adaptive methods for frictionless contact problems. Comput Struct 2001;79:2197-208

[11] Louf F, Combe JP, Pelle JP. Constitutive error estimator for the control of contact problems involving friction. Comput Struct 2003;81:1759-72.

[12] Louf F, Gallimard L, Pelle JP. Un estimateur d'erreur en relation de comportement pour les problèmes d'impact. Rev Eur Mécan Numer 2006;15(6):699-728.

[13] Bostan V, Han W. A posteriori error analysis for finite element solutions of frictional contact problem. Comput Methods Appl Mech Eng 2006;195:1252-74.
[14] Wohlmuth BI. An a posteriori error estimator for two-body contact problems on non-matching meshes. J Sci Comput 2007;33(1):25-45.

[15] Haslinger J, Hlavacek I, Necas J. Numerical methods for unilateral problems in solid mechanics. In: Ciarlet PG, Lions JL, editors. Handbook of numerical analysis, Part 2, vol. IV. North Holland; 1996.

[16] P. Verpeaux, A. Millard, T. Charras, A. Combescure, A Modern Approach of Computer Codes for Structural Analysis, SMIRT 10, Anaheim, 1989.

[17] De Saxcé G. A generalisation of Fenchel's inequality and its applications to the constitutive laws. CR Acad Sci Paris, Ser II 1992;314:125-9.

[18] De Saxcé G, Feng ZQ. The bipotential method: a constructive approach to design the complete contact law with friction and improved numerical algorithms. Math Comput Model 1998;28(N 4-8):225-45.

[19] Prager W, Synge JL. Approximation in elasticity based on the concept of functions space. Quart Appl Math 1947;5:261-9.

[20] Gallimard L. A constitutive relation error estimator based on traction-free recovery of the equilibrated stress. Int J Numer Eng 2009;78:460-82.

[21] Ladevèze P, Pelle JP, Rougeot P. Error estimate and mesh optimization for FE computations. Eng Comput 1991;8:69-80.

[22] Ladevèze P, Pelle JP. Mastering calculations in linear and non-linea mechanics. Mechanical engineering series. Springer; 2005.

[23] Gallimard L, Ladevèze P. Pelle JP. Error estimation and time-space parameters optimization for FEM non-linear computation. Comput Struct 1997;64(1-4):145-56.

[24] Ladevèze P, Moes N. Adaptive control for finite element analysis in plasticity. Comput Struct 1999;73:45-60.

[25] Mandel J. Balancing domain decomposition. Commun Numer Methods Eng 1993;9:233-41.

[26] Dostál Z, Horák D. Theoretically supported scalable FETI for numerical solution of variational inequalities. SIAM J Numer Anal 2007;45:500-13.

[27] Kornhuber R, Krause R. Adaptive multigrid methods for Signorinis problem in linear elasticity. Comput Vis Sci 2002;5:139-48.

[28] Bayada G, Sabil J, Sassi T. A Neumann-Neumann domain decomposition algorithm for Signorini problem. Appl Math Lett 2004;17(10):1153-9. 